\title{
Androgènes et qualité de vie
}

\author{
Sylvain MIMOUN
}

\section{RÉSUMÉ}

Les manifestations cliniques associées au syndrome du déclin androgénique lié à l'âge altèrent la qualité de la vie. Outre les troubles sexuels, des symptômes fonctionnels, des troubles neuropsychiques ainsi que des modifications morphologiques et de l'image de soi participent à cette altération. La testostérone a un impact bénéfique sur la composition corporelle. Son effet anabolisant participe à l'amélioration de la sensation de bien-être par la diminution de la masse grasse, l'augmentation de la masse et de la force musculaire. Des effets directs de la testostérone sur le système nerveux central peuvent aussi participer à l'amélioration de la sensation de bien-être, de la qualité du sommeil et des fonctions cognitives que l'on observe lors de traitements substitutifs. Par contre, des doses supra physiologiques peuvent induire des réactions agressives ou maniaques.

Mots Clés : testostérone, bien-être, anabolisme, système nerveux central

\section{INTRODUCTION}

Il n'y a que relativement peu d'études contrôlées de l'effet du traitement par les androgènes chez l'homme âgé. L'effet bénéfique sur la libido [7,20] s'accompagne d'une sensation de bien-être. Les patients traités en double aveugle reconnaissent le plus souvent la testostérone du placebo [20].

Selon Legros [10], bien que les causes non hormonales, en particulier sociologiques et économiques, rendent plus difficile la démonstration du lien de causalité chez le sujet âgé, l'effet favorable d'un traitement par les androgènes sur certaines plaintes dont "l'irritabilité" et la perte de libido semble cependant bien acquis.

J.L Schlienger [17] a résumé dans un tableau les manifestations cliniques associées au syndrome du déclin androgénique lié à l'âge (Tableau 1). On pourra noter qu'en ce qui concerne la qualité de vie, outre les troubles sexuels, on trouve les symptômes fonctionnels et les troubles neuropsychiques, mais on peut aussi inclure dans le chapitre "qualité de vie", la morphologie et l'image de soi.

En ce qui concerne les modifications de qualité de vie, une partie revient aux modifications de la sexualité, ce qui regroupe tant des facteurs physiologiques que psychologiques.

Outre l'influence du déclin androgénique lié à l'âge, il faut aussi tenir compte des difficultés de nutrition qui peuvent exister chez l'homme plus âgé, du fait, entre autres, de la perte de l'appétit. Le métabolisme de base diminue de 3 à $4 \%$ par décades ce qui induit une diminution de l'énergie chez les hommes âgés. Dans une étude coordonnée par

Correspondance :

Dr. Sylvain MIMOUN, 45 rue Maubeuge, 75009 PARIS 


$\begin{array}{ll}\text { Troubles sexuels : } & \text { - baisse de la libido } \\ & \text { - diminution de l'activité sexuelle } \\ & \text { - fonction érectile médiocre } \\ & \text { - absence d'orgasme } \\ & \text { - éjaculation insuffisante (en qualité et en volume) } \\ & \text { - diminution de la force et de la masse musculaire } \\ \text { Morphologie : } & \text { - dépilation pubienne et axillaire } \\ & \text { - obésité abdominale } \\ & \text { - diminution de volume testiculaire } \\ & \text { - fatigabilité et asthénie } \\ \text { Symptômes fonctionnels : } & \text { - accès d’hypersudation } \\ & \text { - bouffées vasomotrices de type flush } \\ & \text { - troubles du sommeil et insomnie } \\ & \text { - irritabilité ou indifférence } \\ \text { Troubles neuropsychiatriques : } & \text { manque de motivation et d'élan vital } \\ & - \text { troubles de la concentration } \\ & \text { - syndrome anxio-dépressif larvé } \\ & \text { - perte de l'estime de soi } \\ & \text { - troubles de la mémoire récente } \\ \end{array}$

Ritchie et coll. [16] on a trouvé qu'environ $60 \%$ des hommes âgés de 65 ans et plus, vivant en ville, étaient en sous poids.

D'autre part, il faut savoir que la dépression est une maladie commune chez les plus de 65 ans : la dépression sévère touche 1 à $3 \%$ de cette population alors que les dépressions modérées touchent 10 à $15 \%$ d'entre eux. La dépression est souvent sous diagnostiquée et sous-traitée spécialement chez les hommes âgés qui expriment plus souvent des plaintes physiques : «J'ai mal», plutôt que des plaintes psychologiques : «Je suis déprimé».

Quant aux troubles du sommeil, ils font partie des plaintes classiques de l'homme âgé que ceux-ci soient associés ou non à un syndrome dépressif.

Un autre évènement marquant pour la qualité de vie, chez les hommes âgés, c'est la démence qui comprend l'Alzheimer dans plus de $50 \%$ des cas.

J.L Schlienger en conclut que le diagnostic d'un syndrome du déclin androgénique lié à l'âge doit aboutir logiquement à la proposition d'un traitement substitutif dont le but est de restaurer libido et activités sexuelles satisfaisantes, et sensation de bien-être [17]. Mais un tel traitement n'est acceptable que si les bénéfices observés sont bien supérieurs aux risques éventuels, sous réserve d'une évaluation objective qui manque encore cruellement dans la littérature médicale [4].

\section{QUALITÉ DE VIE ET EFFET ANABOLISANT DES ANDROGÈNES}

D'après la revue de la littérature faite par G. Reyes-Ortega et coll. [15], les effets déjà connus du traitement androgénique chez le sujet adulte comprennent, entre autres, l'augmentation de la masse musculaire, la diminution de la masse adipeuse et l'amélioration du bien-être.

De nombreuses études ont montré que la testostérone a un impact bénéfique sur la composition corporelle.

Morley en 1993 [12] a étudié l'administration de testostérone chez le sujet âgé avec hypogonadisme (testostérone biodisponible inférieure à $75 \mathrm{ng} / \mathrm{dl}$ ) : 14 hommes de moyenne d'âge de 77 ans ont reçu un traitement, soit placebo (6 malades) soit testostérone ( 8 malades) : $200 \mathrm{mg}$ tous les 15 jours, pendant 3 mois. Parmi les résultats les plus importants dans le groupe testostérone, on trouve l'augmentation de la force du poignet. Il n'y a eu aucune action sur le poids ou même l'humeur, cependant la sensation de bien-être a été accrue très significativement.

Bashin et coll. [2] ont administré $600 \mathrm{mg}$ de testostérone/semaine pendant 10 semaines à des hommes répartis en 4 groupes, Sans exercice-Placebo $(n=10) /$ Sans exercice-Testostérone $(n=10) /$ Exercice-Placebo $(n=9) /$ Exercice-Testostérone $(\mathrm{n}=11)$.

Finalement, on constate une augmentation de poids, une augmentation non significative de la masse grasse et une 
augmentation très significative de la masse maigre (triceps et quadriceps), sauf dans le groupe placebo sans exercice.

De façon plus remarquable encore, l'augmentation de la force musculaire a été plus importante chez les sujets du groupe "exercice + testostérone", mais elle est également présente chez les sujets sous testostérone sans exercice.

Ainsi, l'effet anabolisant de la testostérone se produit aussi bien chez les hommes hypogonadiques traités avec des doses physiologiques de testostérone que chez les hommes eugonadiques qui ont reçu des doses pharmacologiques de testostérone [2]. Snyder et coll. [18], ont attiré notre attention sur le fait que les études de l'administration d'androgènes aux hommes âgés ont montré une augmentation constante de la masse maigre mais une augmentation variable de la force musculaire.

Aucune de ces études n'a montré de corrélation entre le changement de la force musculaire et la performance fonctionnelle.

Cependant, notons que toutes ces études d'androgénothérapie ont été faites exclusivement chez les hommes âgés et en bonne santé. Il reste donc à voir quel impact l'administration de la testostérone pourrait avoir chez un homme âgé fragile qui a en général un taux de testostérone de 10 à 15 $\%$ plus bas que l'homme en bonne santé ainsi que l'ont souligné Gray et coll. [5]. Il est possible que cette population soit celle qui tirerait le plus de bénéfices d'un traitement de la testostérone. Il serait particulièrement intéressant de voir si les changements au niveau de la force musculaire dans ce groupe se traduiraient par une augmentation de l'activité fonctionnelle et potentielle ainsi que par la diminution des chutes et des fractures.

\section{QUALITÉ DE VIE ET EFFET CÉRÉ- BRAL DES ANDROGÈNES}

Au niveau du système nerveux central humain, la réponse la plus importante de l'administration de la testostérone est l'amélioration du bien-être par des mécanismes qui nous sont encore inconnus, peut-être d'action centrale, liés aux neurotransmetteurs, ou liés à l'axe GH-IGF-1 [11, 22].

L'amélioration du bien-être chez l'adulte avec hypogonadisme est évidente jusqu'à l'obtention des niveaux physiologiques de testostérone. Apparemment l'augmentation de la dose au-delà de ce taux ne produit pas une amélioration de la réponse. Quant aux modifications du comportement (agressivité), elles n'ont pas été corrélées à l'administration de testostérone même à dose supraphysiologique selon Tricker et coll. [21]. Ces résultats semblent contradictoires avec des études précédentes : Perry et coll. [13] ont montré que les utilisateurs de stéroïdes ont une augmentation d'hostilité, d'anxiété, de somatisation et de dépression.
Hannan et coll. [8] ont observé une augmentation significative de l'hostilité et du sous-groupe ressentiment/agression du Minnesota Multiphasic Personality Inventory après 6 semaines de traitement avec de la testostérone ou le stéröde anabolisant, la nandrolone.

Dans une étude en double aveugle Su et coll. [19] ont comparé la methyltestotérone contre placebo, durant deux semaines en faisant des essais séquentiels auprès de 20 hommes volontaires sains. Tous les trois jours ces hommes ont pris consécutivement un placebo (état initial), puis de petites doses de méthyltestotérone $(40 \mathrm{mg} / \mathrm{j})$, de fortes doses de méthyltestotérone $(240 \mathrm{mg} / \mathrm{j})$ et à nouveau un placebo (fin de traitement). Il ressort de cette étude que les fortes doses ont entraîné des augmentations significatives, bien que faibles sur l'humeur positive (euphorie, énergie et excitation sexuelle), mais aussi sur l'humeur négative (irritabilité, humeur changeante, sentiments violents, colère et hostilité), et les attitudes et comportements inhibiteurs (être distrait, oublis et confusion). Un état maniaque a été observé chez l'un des sujets, ce qui représente $5 \%$ de la population étudiée, et un autre devint hypomaniaque.

Les études sur les utilisateurs illicites de stéroïdes anabolisants-androgèniques suggèrent que certains développent des réactions agressives ou maniaques. Cependant les évaluations contrôlées de ces effets restent limitées. Pope et coll. [14] ont effectué une étude randomisée en double aveugle, contre placebo auprès de 56 hommes âgés de 20 à 50 ans avec des doses progressives (allant de $150 \mathrm{mg} / \mathrm{sem}$ à $600 \mathrm{mg} / \mathrm{sem}$ ) de cypionate de testostérone pendant 6 semaines $(150 \mathrm{mg} / \mathrm{sem}$ les deux premières semaines, 300 $\mathrm{mg}$ les troisième et quatrième semaines et $600 \mathrm{mg} / \mathrm{sem}$ les deux dernières semaines). Les résultats des questionnaires psychiatriques ont montré que le traitement à la testostérone augmentait significativement les scores maniaques du «Young Mania Rating Scale» YMRS ( $\mathrm{p}=0,002)$, les scores maniaques $(p=0,003)$ et les réponses agressives $(p=0,03)$ sur le «Point Substraction Agression Paradigm». Les réponses à la testostérone ont été hautement variables : sur les 50 participants qui ont reçu $600 \mathrm{mg} / \mathrm{sem}$ de cypionate de testostérone, 42 (84\%) ont montré des effets psychiatriques minimaux (un score d'YMRS maximum : $<10$ ), six $(12 \%)$ sont devenus moyennement hypomaniaques (score d'YMRS : 10-19) et deux (4\%) sont devenus nettement hypomaniaques (score d'YMRS : > 20).

On peut se demander ce qu'il convient de préconiser chez les hommes plus âgés : un traitement substitutif chez le patient avec un hypogonadisme évident, un traitement à dose physiologique chez tous les hommes âgés ou bien un traitement pharmacologique supraphysiologique. La réponse peut être différente chez l'homme âgé "normal" ou chez les grands malades (polypathologie, dénutrition grave, état de choc). 
Une situation pouvant être informative est le développement de l'indication du traitement androgénique dans les maladies, telles que les patients infectés par le VIH.

Comme l'ont souligné Grinspoon et coll. [6], l'hypogonadisme est prévalent chez les hommes infectés par le VIH ce qui réduit significativement leur qualité de vie et des perturbations de l'humeur ont été signalées. Cette étude (sur 52 hommes hypogonadiques et 10 eugonadiques qui étaient infectés par le VIH) a montré que l'administration de testostérone a entraîné une amélioration significative sur les scores de dépression. Cet effet peut être un effet direct de la testostérone sur le poids et sur d'autres indices anthropométriques. D'autres études sont cependant nécessaires pour pouvoir affirmer les effets de la testostérone sur les signes cliniques de la dépression chez les patients infectés par le VIH.

\section{DES EFFETS COMPORTEMENTAUX FAVORABLES.}

L'administration d'androgènes a des effets comportementaux globalement favorables selon Wang [22]. Elle améliore la vigueur sexuelle et la sensation de bien-être des sujets hypogonadiques bien substitués. Il existe de surcroît une amélioration de la qualité du sommeil, une diminution de la fatigue. La présence de récepteurs à la testostérone dans l'hippocampe et autour du cortex médiotemporal, zones impliquées dans la mémoire spatiotemporale, explique peut-être l'amélioration des fonctions cognitives rapportées dans plusieurs études. A l'inverse, administrer en excès de la testostérone favorise l'agressivité, l'hypersexualité et le passage à l'acte délictueux. De tels inconvénients n'ont pas été rapportés dans le cadre d'une substitution hormonale justifiée et adéquate.

\section{CONCLUSION}

Pour Bagatell et Bremner [1], l'androgénothérapie est un moyen raisonnable de corriger un déficit dûment établi par la coexistence de symptômes fonctionnels, d'anomalies physiques et d'une hypotestostéronémie franche.

Dans son éditorial, Hayes [9], souligne l'intérêt pharmacocinétique de la voie transcutanée pour l'administration de testostérone et plus particulièrement du gel hydroalcoolique de testostérone qui permet d'atteindre des concentrations physiologiques de testostérone en 30 minutes avec des valeurs stables ensuite, il augmente les fonctions sexuelles, la masse musculaire et la force musculaire et diminue la masse grasse chez les hommes hypogonadiques et est mieux toléré que les patchs.

Débuté à bon escient, le traitement doit être poursuivi jusqu'à ce que survienne l'une des situations de contreindication ou de non-indication dès lors que les objectifs thérapeutiques sont atteints. L'absence d'efficacité du traitement doit remettre en cause son bien-fondé car seule la réversibilité des anomalies justifie le traitement $[3,7]$.

\section{RÉFÉRENCES}

1. BAGATELL C.J., BREMNER W.J. : Androgens in men-uses and abuses. N. Engl. J. Med., 1996, 334 : 707-714.

2. BHASIN S., STORER T.W., BERMAN N., et al. : The effects of supraphysiologic doses of testosterone on muscle size and strength in men. N. Engl. J. Med., 1996, $335: 1-7$.

3. BHASIN S., BAGATELL C.J., BREMNER W.J., et al. : Issues in testosterone replacement in older men. J. Clin. Endocrinol. Metab., 1998, 83 : 3435-3448.

4. BARDIN C.W., SWERDLOFF R.S., SANTEN R.J. : Androgens: risks and benefits. J. Clin. Endocrinol. Metab., 1991, 73 : 4-7.

5. GRAY A., FELDMAN H.A., Mc KINLAY J.B., LONGCOPE C. : Age, disease and changing sex hormone levels in midle-aged men : results of the Massachusetts male aging study. J. Clin. Endocrinol. Metab., 1991, 73 : 1016-1025.

6. GRINSPOON S., CORCORAN C., STANLEY T., BAAJ A., BASGOZ N., KLIBANSKI A. : Effects of hypogonadism and testosterone administration on depression indices in HIV-infected men. J. Clin. Endocrinol. Metab., 2000, 85 : 60-65.

7. HAJJAR R.R., KAISER F.E., MORLEY J.E. : Outcomes of longterm testosterone replacement in older hypogonadal males : A retrospective analysis. J. Clin. Endocrinol. Metab., 1997, 82 : 3793-3796.

8. HANNAN C.J. Jr., FRIEDL K.E., ZOLD A., KETTLER T.M., PLYMATE S.R. : Psychological and serum homovanillic acid changes in men administered androgenic steroids. Psychoneuroendocrinology, 1991, 16 : 335-343.

9. HAYES F.J. : Testosterone-fountain of youth or drug of abuse? J. Clin. Endocrinol. Metab., 2000, 85 : 3020-3023.

10. LEGROS J.J. : Vers un consensus pour le traitement androgénique substitutif de l'andropause. Rev. Med. Liège, 2000, $55: 449-453$.

11. MORALES A.J., NOLAN J.J., NELSON J.C., YEN S.S. : Effects of replacement dose of dehydroepiandrosterone in men and women of advancing age. J. Clin. Endocrinol. Metab., 1994, 78 : 1360-1367.

12. MORLEY J.E., PERRY H.M. 3rd, KAISER F.E., KRAENZLE D. et al. : Effects of testosterone replacement therapy in old hypogonadal males : a preliminary study. J. Am. Geriatr. Soc., 1993, $41: 149-152$.

13. PERRY P.J., ANDERSEN K.H., YATES W.R. : Illicit anabolic steroid use in athletes. A case series analysis. Am. J. Sports Med., $1990,18: 422-428$.

14. POPE H.G. Jr, KOURI E.M., HUDSON J.I. : Effects of supraphysiologic doses of testosterone on mood and aggression in normal men : a randomized controlled trial. Arch. Gen. Psychiatry, 2000, $57: 133-140$.

15. REYES-ORTEGA G., NOURHASHEMI F., VELLAS B., ALBAREDE J.L. : Traitement androgène chez le sujet agé, revue de la littérature. L'année gérontologique, 1997, 11 : 377-389.

16. RITCHIE C.S., BURGIO K.L., LOCHER L.J., et al. : Nutritional status of urban homebound older adults. Am. J. Clin. Nutr., 1997, $66: 815-818$. 
17. SCHLIENGER J.L. : L'andropause existe : mais comment la traiter ? Rev. Prat., 2001, $15: 277-281$.

18. SNYDER P.J., PEACHEY H., HANNOUSH P. et al. : Effect of testosterone treatment on body composition and muscle strength in men over 65 years of age. J. Clin. Endocrinol. Metab., 1999, 84 : 2647-2653.

19. SU T.P., PAGLIARO M., SCHMIDT P.J., PICKAR D., WOLKOWITZ O., RUBINOW D.R. : Neuropsychiatric effects of anabolic steroids in male normal volunteers. J. Am. Med. Ass., 1993, $269: 2760-2764$.

20. TENOVER J.S. : Effects of testosterone supplementation in the aging male. J. Clin. Endocrinol. Metab., 1992, 75 : 1092-1098.

21. TRICKER R., CASABURI R., STORER T.W., et al. : The effects of supraphysiological doses of testosterone on angry behavior in healthy eugonadal men-a clinical research center study. J. Clin. Endocrinol. Metab., 1996, 81 : 3754-3758.

22. WANG C., ALEXANDER G., BERMAN N., et al. : Testosterone replacement therapy improves mood in hypogonadal men-a clinical research center study. J. Clin. Endocrinol. Metab., 1996, 81 : 3578-3583.

\section{ABSTRACT \\ Androgens and quality of life.}

Sylvain MIMOUN

Clinical manifestations of androgenic deficiency in the ageing male induce deterioration of quality of life. In addition to sexual disorders, functional and neuropsychiatric symptoms, and morphological changes affecting the self-image participate in this deterioration. Testosterone has a beneficial impact on body composition. The anabolic effects of testosterone help to improve the feeling of well-being by reducing fat mass, and increasing muscle mass and strength. The direct effects of testosterone on the central nervous system may also participate in the improvement of the feeling of wellbeing, the quality of sleep and cognitive functions, as observed during hormone replacement therapy. However, administration of supraphysiological doses of testosterone can induce aggressive reactions and manisc episodes.

Key Words: testosterone, well-being, anabolism, central nervous system 